\title{
A joint longitudinal and survival model for dynamic treatment regimes in Presence of Competing Risk Analysis
}

\author{
Atanu Bhattacharjee \\ Centre for Cancer Epidemiology, The Advanced Centre for Treatment, Research and Education in Cancer (ACTREC), Tata Memorial Centre, Navi Mumbai, 410210, India
}

\section{A R T I C L E I N F O}

\section{Keywords:}

Dynamic treatment regime

Competing risk

Sequential rule

Personalized medicine

Bayesian

\begin{abstract}
A B S T R A C T
Background: In oncology setup, the personalized medicine with the Dynamic Treatment Regime(DTR) is the attractive tool for treatment management. It is based on sequence rule by changing the required treatment by looking into patients dynamic condition repeatedly. This repeatedly measured condition generates the longitudinal data and the time-to-event data jointly. The time-to-event data is generated with competing risks. Now handling the joint longitudinal and survival data with competing risks is itself challenging. It becomes more challenging to decide the best effective treatment strategy while we work with DTR approach in presences of competing risks through joint longitudinal and survival model.

Methods: This article is dedicated towards development of statistical methodology to handle competing risk analysis for DTR in repeatedly measured survival data. In this study a simulated dataset is used to resemble the observed data distribution seen in a motivating cancer trial data.

Results: This algorithm is prepared through Bayesian analysis to obtain the best effective therapeutic regimen. The OpenBUGS function is prepared, which provides the therapeutic effect comparison and there after estimations between different treatment sequences.

Conclusion: This developed method is easy to handle for personalized medicine context in oncology for supportive decision rule.
\end{abstract}

\section{Introduction}

In oncology research, the primary aim is to observe the death rate and thereafter make a conclusion about best effective treatment. However, patients under study may die due to other causes as well. The death of the patients due to other causes is defined as competing-risk. There is a strong literature existing on competing risk analysis.

The competing risk analysis is a part of survival analysis. It is the fact that the survival analysis is the widely adopted tool in oncology research. There is unlimited literature on survival analysis through the parametric, semiparametric and nonparametric approaches. ${ }^{1-5}$ Generally, in oncology setup patients are followed for certain time point and thereafter the occurrences of death are measured through the effect of time-dependent covariates. The time-dependent covariates are measured through longitudinal or follow-up measurements. Now the statistical model to work together with repeatedly measured time-dependent covariates and survival event known as joint longitudinal and survival modeling. ${ }^{6}$ There is some recent attempt towards the development of statistical methods on joint longitudinal and survival modeling ${ }^{7-11}$

In oncology research, there is high chance that one treatment may fail to respond or becomes resistance in due course of the study period. An alternative choice is to try an alternative therapy to the resistant patients. These are known as second-line therapy. Now repeated applications of different types of therapy after resisting with one treated therapy exposed the patients towards multiple therapies. But the permanent event of the life of a patient's i.e. death is obvious to a fatal disease like cancer. Now blaming one specific therapy among different lines of therapy becomes difficult. These challenge provides the scope of development of statistical methodology towards dynamic treatment regime and widely known as DTR survival analysis. ${ }^{12-17}$ There is very limited and recent development in DTR approaches. During one routine work with survival analysis of lung cancer trial data, we faced a challenge to work with repeatedly measured survival data having competing risk information. There were several lines of therapy to attempt the best effective therapy in lung cancer patients. We searched the existing literature on Joint Longitudinal and Survival Model for Dynamic Treatment Regimes in Presence of Competing Risk Analysis. But unfortunately, we failed to get anything. Particularly we were interested to get the statistical foundation to serve our problem. But we did not even get any theoretical work towards our faced problem. It inspired us to develop an application tool towards Joint Longitudinal and

E-mail address: atanustat@gmail.com. 
Survival Model for DTR in Presence of Competing Risk Analysis. While we attempted to apply our developed method to lung cancer data, we did not get ethical approval due to some stringent regulation. In this situation, a simulation study is performed to mimic the observed data to generate the similar scenario that observed in real lung cancer patients data. The objective of this work is served through the development of a statistical method for joint modeling in longitudinal and survival analysis with DTR in presence of competing risk.

In conventional survival analysis, each patient is followed until the single type of event is occurred. The presences of more than one event are known as competing risks. The hazard plays a predominant role in estimation in presences of left truncated or censored data. Three different hazards can be discriminated in presences of competing risk setting. However, the occurrence of the marginal hazard of the event protected the occurrence of other types of an event. ${ }^{18}$ The marginal modeling is only applicable while the distribution time of the event of interest is independent in nature. However, it is not feasible to test the presences of independence. ${ }^{19}$ The testing the independence assumption can be avoided through the application of cause-specific hazard. It assumed that the events due to other causes as censored in nature. Contrary to the standard survival setting with only one type of event, it is not required to link one-to-one relation between covariates and causespecific hazard rate. The alternative is to perform the subdistribution hazard model. ${ }^{20}$

Joint analysis of longitudinal measurements and survival time is found suitable to assess the factors effects. ${ }^{8,21}$ The existing difficulties in Cox Proportional hazards model related to missing time-varying covariates and measurement error can be smoothly handled by joint modeling. ${ }^{2-26}$ All the authors applied the common assumption as noninformative censoring and in presences of single failure type, the noninformative censoring is only useful. But in the presence of competing risks, the noninformative censoring is not appropriate. Recently, there is an attempt towards the development of joint longitudinal and survival model in presences of multiple failure types. ${ }^{27}$ The longitudinal portion is explored through linear mixed effect submodel and survival component through frailty model. These two submodels are linked through latent random effect models. This is to be noted that the competing risks analysis through cause-specific proportional hazard assumption treats the only one risk as the event of interest at a time and others as noninformative censoring events. ${ }^{5,28,29}$ But it stratified the data at any point of competing risk analysis.

The framework of Dynamic Treatment Regime (DTR) is proposed by Murphyon mean response modeling. ${ }^{30}$ It is also applied through optimal response modeling ${ }^{31}$ and nested model on DTR by Robins. ${ }^{32}$ The development of DTR opens the scope towards personalized medicine. Very careful formulation and assumptions are required for valid inference to apply DTR. There are several estimation procedures known as G-estimation method, ${ }^{33}$ Q-learning method and A learning procedure. ${ }^{34-36}$ However, all these decision-making algorithms are computer incentive. It is possible to measure the required optimum time for second-line therapy. ${ }^{37}$ It is observed with good estimator by penalized least square method. ${ }^{38}$ However, there is the very limited extension of DTR in the survival context. The progress in survival context is documented with marginal modeling ${ }^{30}$ and inverse weighting method ${ }^{39,40}$ and the extension are proposed by weighted risk set estimator. ${ }^{41}$ In nonparametric setup, the extension over Nelson-Aalen estimator is proposed by weighted risk set estimator. ${ }^{41}$

\subsection{Generated data set}

The approach with DTR is relatively new and other challenges like competing risks to handle DTR dataset are not even documented. We failed to get any real life data to work and illustrate towards development of methodology on DTR competing risk for joint longitudinal and survival model. But recently in our routine departmental data services a retrospectively observed dataset observed with presences of repeatedly measured covariates and survival events with dynamic treatment regime. Due to some ethical constraint in that dataset, we could not be used that data in this analysis. However, in this study a similar dataset are generated to mimic with observed dataset. The dataset is generated to resemble the observed data distribution seen in a motivating Lung Cancer trial. A total of six lines therapy are generated through the assumption of binomial distribution having sample size 300 and the lines of therapy are defined as $A_{1}, A_{2}, A_{3}, A_{4}, A_{5}$ and $A_{6}$ arms respectively. The binomial distributions of these six lines of therapy are generated independently. The six lines of therapy are generated independent to provide priority to the intention to treat therapy than randomized trial. Because in routine clinical practice, clinician do avoid the randomization schedule to select any treatment. It is always preferable to prepare statistical methodology towards opportunity to select best effective dose to the clinician. The statistical methodology generated through the platform with randomized trial may not be appropriate for clinician to accept due to the limitation with reproducibility. Because, in routine practice the randomization schedule is always avoided. It is considered that any treatment between $A_{1}$ to $A_{6}$ can be preferred by any clinician at any point of time for any subject. The dataset are generated like there are 66 patients to whom $A_{2}$ considered as first choice over $A_{1}$. The presence of any treatment is defined as 1 otherwise 0 . The condition is provided as $\sum_{i=1}^{6} A_{i} \geq 1$. A total of 160 patients are generated with $A_{1}$ as first choice of therapy followed by 158,159,162,155 and 162 for $A_{2}, A$ ${ }_{3}, A_{4}, A_{5}$ and $A_{6}$ respectively. The survival duration is obtained from normal distribution with median duration of 24 months. The death and competing status is generated through the rbinom(300,2,.03) function in R.A total of 161 subjects are generated with censored, 117 and 22 as died and competing risks event respectively. The "CumIncidence" function available at ${ }^{36}$ is used to generate cumulative incidence.

The descriptive presentation on different treatment groups with censored, death and competing risks are given in Table 1 . The estimates obtained with competing risks are presented in Table 2 . The proposed survival function is used to formulate the estimates with DTR. In this survival function, the survival duration is multiplied with a corresponding weight of each treatment $\left(A_{i}, i=1\right.$ to 6$)$. It is assumed that each patient spend his each unit of survival/follow-up time with a particular arm i.e $A_{1}$ to $A_{6}$. Now the patient spend maximum time with a treatment is assigned as a maximum weight and followed by other treatments according to their time spend. If any of this treatment $A_{1}$ to $A_{6}$ is not assigned to patients then corresponding weight will be zero for survival function. The unique feature of DTR is that one patient is expected to have exposed to different treatments sequentially, and all the treatment can be allocated randomly in any sequence. But the treatment assigned lastly and death occurred thereafter should be penalized. There is limitation of existing statistical methodology. However, this proposed survival function can taken care of it. This random sequence allocation is considered in the context of random variable data generation purpose only. In real life scenario, it is expected that treatment allocation in any sequence will be based on clinical evidence and experience of a clinician. It should not be based on blind randomization sequentially.

\subsection{Statistical model}

Let the sample size of any study is $\mathrm{n}$. The duration of survival is defined with function $S\left(t_{i j k}\right)$.

Table 1

Treatment wise distribution of death, competing risk and censored case.

\begin{tabular}{llllllll}
\hline \multirow{2}{*}{ Treatment } & Cases & Death & Censored & Treatment & Cases & Death & Censored \\
\hline$A_{1}$ & 160 & 85 & 13 & $A_{4}$ & 162 & 84 & 15 \\
$A_{2}$ & 158 & 83 & 13 & $A_{5}$ & 155 & 82 & 13 \\
$A_{3}$ & 159 & 83 & 13 & $A_{6}$ & 162 & 57 & 14 \\
\hline
\end{tabular}


Table 2

Treatment wise hazard ratio estimates with treatments as time-dependent covariates.

\begin{tabular}{llllll}
\hline Treatment & $\begin{array}{l}\text { Hazard } \\
\text { ratio(SD) }\end{array}$ & $\begin{array}{l}95 \% \\
\text { Credible } \\
\text { Interval }\end{array}$ & Treatment & $\begin{array}{l}\text { Hazard } \\
\text { ratio(SD) }\end{array}$ & $\begin{array}{l}95 \% \\
\text { Credible } \\
\text { Interval }\end{array}$ \\
\hline$A_{1}$ & $2.82(1.18)$ & $(2.11,4.09)$ & $A_{4}$ & $2.18(1.11)$ & $(1.76,2.82)$ \\
$A_{2}$ & $2.43(1.11)$ & $(1.99,3.09)$ & $A_{5}$ & $2.11(1.24)$ & $(1.49,3.63)$ \\
$A_{3}$ & $3.49(1.37)$ & $(2.09,7.38)$ & $A_{6}$ & $3.38(1.39)$ & $(2.01,7.38)$ \\
\hline
\end{tabular}

Further, it is assumed that the $k t h$ order treatment is $j$ th given to an ith individual.

The survival function is formulated as

$S\left(T_{i}\right)=\sum_{j=1}^{6} \sum_{k=1}^{6} S\left(T_{i j k}\right), j=1$ to 6 and $k=1$ to 6

The methodology is proposed with 6 lines of maximum therapy(j) to a patients in his/her entire course of treatment and order(k) of treatment is maximize with 6 is defined for $S\left(T_{i}\right)$ as

$\widehat{\mathrm{S}}\left(\mathrm{T}_{\mathrm{ijk}}\right)=\sum_{\mathrm{k}=1}^{6} \frac{\mathrm{k} * \mathrm{~S}\left(\mathrm{~T}_{\mathrm{ijk}}\right)}{\sum_{\mathrm{i}=1}^{6} \sum_{\mathrm{j}=1}^{6} \sum_{\mathrm{k}=1}^{6} \mathrm{~S}\left(\mathrm{~T}_{\mathrm{ijk}}\right)}$

$\hat{S}\left(T_{j}\right)=\frac{S\left(T_{i j}\right)}{\hat{S}\left(T_{i j k}\right)}$

The variance is estimated as

$$
\widehat{\operatorname{Var}}\left(\hat{S}\left(T_{j}\right)\right)=\hat{S}\left(T_{j}\right) \sum_{i, t_{i} \leq t} \frac{d_{i j}}{n_{i j}\left(n_{i j}-d_{i j}\right)}
$$

This study is dedicated towards development of joint longitudinal and survival model. The repeatedly measured covariates is defined as $x_{i t}$. The ith individuals $j$ th time point measured covariate is $x_{i t}$. The cause of death is defined as $m=1,2$. The term $m=1$ represents that cause of death is due to cancer or $\mathrm{m}=2$ represents death due to competing risk.

The hazard function is presented as

$\lambda_{m}\left(t_{j k}, x_{i t}\right)=\lim _{d t_{j k} \rightarrow 0} \frac{\operatorname{Pr}\left\{t_{j k} \leq T<t_{j k}+d t_{j k}, M=m \mid T_{j k} \geq t_{j k}, x_{i t}\right\}}{d t}$

The conditional probability that subject will die due the covariates $x_{i}$ within the interval $\left[t_{j k}, t_{j k}+d t_{j k}\right]$ will be calculated by m-th cause of death, given that the subject is alive before the time $t_{j k}$. The probability into a rate is considered by dividing by $\mathrm{dt}$ and then take the limit as $d t_{j k}$ $\rightarrow 0$.

$\lambda\left(t_{j k}, x_{i t}\right)=\sum_{m=1}^{2} \lambda m\left(t_{j k}, x_{i t}\right)$

Further, the competing risk density function is called to serve two state i.e. death due to disease or death due to other causes with the function of $\mathrm{t}$ as

$f(t)=\frac{P\left\{t_{j k} \leq T<t_{j k}+d t_{j k}, M=m \mid x\right\}^{r}}{d t_{j k}}$

This function considered the unconditional of risk to die at time $t$ due to $\mathrm{m}$ causes. The complete density function to serve the death causes is presented as

$f(t, x)=\sum_{i=1}^{m} \sum_{j=1}^{6} \sum_{k=1}^{6} f\left(t_{j k}, x\right)$

Now considering the competing risk with DTR, It is required to formulate the regression model. The likelihood function is defined as
$L=\prod_{i=1^{\prime}}^{n} \lambda_{m}\left(t_{i}, x_{i}\right)^{d_{i}} S\left(t_{i}, x_{i}\right)$

The survival probability is defined by $S\left(t_{i}, x_{i}\right)$.

Now, $S\left(t_{i}, x_{i}\right)=\prod S_{m}\left(t_{i}, x_{i}\right)$

The survival function is formulated as

$S\left(t_{i}\right)=\exp \left(-t_{i}\right)$

The follow-up times are ordered as $0<t_{1}<t_{2}<\ldots<t_{N}$.

The orders are prepared with distinct point of time by $T$. The risk set is formulated as $R\left(t_{p}\right)$, for the time point $t_{p}$. The individual at risk at a time point $t_{p}$ is denoted as $n_{p}$. The number of event occurrence is $d_{p}$. The time span spend by individual is assumed as discrete. The probability of occurrence of event at $t_{p}$ is

$\lambda\left(t_{p}\right)=P\left(T=t_{p} \mid T>t_{(p-1)}\right)$

Now the term $\lambda\left(t_{p}\right)$ can be represented as

$\lambda\left(t_{\mathrm{p}}\right)=\mathrm{dp} / \mathrm{np}$

The probability of surviving at time $\mathrm{t}$ is

$\widehat{S}\left(t_{i p}\right)=\widehat{S}\left(t_{i p-1}\right)\left(1-\left(\lambda\left(\widehat{t_{i p j}}\right)\right)\right)$

$\widehat{S}\left(t_{i p}\right)=\widehat{S}\left(t_{i p-1}\right)\left(1-\frac{d_{i p}}{n_{i p}}\right)$

$\widehat{S}(t)=\prod_{i p: t_{j} \leq t}\left(1-\frac{d_{i p}}{n_{i p}}\right)$

While the sample population i.e. $n_{p}$ starts to incline then the estimates obtained through Kaplan-Meier becomes continuous. The covariates of interest $(Z)$ is defined as

$\lambda(t \mid z)=\lambda_{0}(t) \exp \left(\beta^{T} z\right)$

The regression coefficient for the covariates of interest $(\beta)$ on baseline $\left(\lambda_{0}(t)\right)$ hazard function is defined as

$L(\beta)=\prod \frac{\exp \left(\beta^{T} z_{l}\right)}{\sum \exp \left(\beta^{T} z_{l}\right)}$

The cause of failure (D) is generated by

$\lambda_{m}(t)=\lim _{t \rightarrow 0} \mathrm{P}\left(t \geq T<t+\Delta t_{t}, D=m \mid T \geq t\right)$

is

$\lambda_{m}\left(t_{j}\right)=P\left(T=t_{i p}, D=m \mid T>t_{i p-1}\right)$

It is estimated as,

$\hat{\lambda}_{m}\left(t_{i p}\right)=\frac{d_{i p}}{n_{i p}}$

The probability of death due to mcauses at a specific time is

$S\left(t_{i}\right)=\Pi_{i p=t_{p}<t}\left(1-\sum_{m=1}^{M} \widehat{\lambda_{m}}\left(t_{i p}\right)\right)$

The covariates $(\mathrm{Z})$ linked regression coefficient $\left(\beta_{m}\right)$ for $\mathrm{m}$ cause of death is defined as

$\lambda_{m}(t \mid Z)=\lambda_{m, 0}(t) \exp \left(\beta_{m}^{z} Z\right)$

\subsection{Analysis}

The proposed method is open to adopt for routine clinical practice not selected for randomly selected treatment arm. The competing risk is performed with conventional method and Bayesian Counterpart 
Table 3

Posterior estimates of the parameters observed through competing risk analysis.

\begin{tabular}{lllll}
\hline \multirow{2}{*}{ Treatment } & \multicolumn{2}{l}{ Death due to treatment Failure } & \multicolumn{2}{l}{ Death due to Competing Risk } \\
\cline { 2 - 5 } & Hazard & 95\% Credible & Hazard & 95\% Credible \\
\cline { 2 - 5 } & Ratio(SD) & Interval & Ratio(SD) & Interval \\
\hline$A_{1}$ & $1.82(3.18)$ & $(0.21,20.90)$ & $2.82(1.18)$ & $(2.11,4.09)$ \\
$A_{2}$ & $1.16(1.91)$ & $(0.32,4.34)$ & $2.43(1.11)$ & $(1.99,3.09)$ \\
$A_{3}$ & $0.95(1.05)$ & $(0.82,1)$ & $3.49(1.37)$ & $(2.09,7.38)$ \\
$A_{4}$ & $1.16(1.91)$ & $(0.32,4.34)$ & $2.18(1.11)$ & $(1.76,2.82)$ \\
$A_{5}$ & $0.00(32.45)$ & $(0.00,0.11)$ & $2.11(1.24)$ & $(1.49,3.63)$ \\
$A_{6}$ & $1.02(1.05)$ & $(0.95,1.16)$ & $3.38(1.39)$ & $(2.01,7.38)$ \\
\hline
\end{tabular}

separately and corresponding results are provided with Tables 1 and 2 . As an alternative the Bayesian counterpart is also extended to strengthen the data analysis section by considering covariates in competing risk. The Bayesian likelihood defined is considered to work with competing risk with covariates. The covariates in the model is defined as $x_{i}$ for treatment. The Bayesian computation is performed through open source software OpenBUGS(www.openbugs.net). A total of 20,000 iteration are performed with markov chain monte carlo(MCMC) with a separate refreshment of 100 at a time. The posterior mean(SD) are generated with $95 \%$ credible interval and presented in Table 2 . The representation of six different arms are defined with $\beta_{i 1}, i=1$ to 6 to represent the death due to cancer not for competing risks. The representation on competing risk are presented with Table 3. Any factor contributing of death or competing risk can be represented through $95 \%$ credible intervals for each categories. The hazard ratio measured below one for a specific treatment can be defined as effective treatment towards prolonging the survival. In Table 3 the estimated hazard ratio for $A_{3}$ is estimated as 0.95 with credible interval $0.82,1$ ) for death due to treatment failure of individuals. The therapeutic arm $A_{3}$ can be defined as best therapeutic arm.

\section{Discussion}

The inference draw in the presences of DTR is really challenging. The parameters are not regular underlying longitudinal data distribution and it badly affects the estimates. The DTR is approached to estimate the parameter through optimal principle. This study is dedicated towards development of statistical methodology to deal with competing risks in DTR, whereas data is observed with joint longitudinal and survival setup. This is the first approach towards combined work with DTR and competing risk. The data analysis is performed with the conventional methodology to deal with competing risk and DTR separately. Due to an absence of conventional combined statistical methodology to work with joint longitudinal and survival modeling with DTR in competing risk analysis, this data analysis is performed separately with the conventional methodology to deal with competing risk and DTR. Finally, our proposed combined method with DTR and competing risk is also performed for statistical inference. It is the fact that the presences of competing risks are equally important for oncology research. However, it is an overlooked area in routine oncology research analysis. ${ }^{43,44}$ It is required to test the assumption about failure time distribution and shape and scale parameter of survival distribution. ${ }^{45}$ It is concluded that Bayesian counterpart in competing risk setting is more informative than the conventional methods. ${ }^{29,46-48}$ There are some statistical compatible to handle the competing risk with the conventional method. ${ }^{42,49}$ The dedicated $\mathrm{R}$ packages like mstate and timereg are suitable to work with competing risk in the simpler way. However, there is not any available function to work present our challenge in statistical analysis. The prepared code in OpenBUGS is available on request by email to the corresponding author. Moreover, this proposed methods can be used in routine data analysis having presences of DTR and competing risk. It is expected that this proposed method will help towards treatment decision in personalized medicine.

\section{Conclusion}

The occurrence of death due to other causes in oncology research is practical. So the competing risk analysis cannot avoid. Similarly, over the decade's oncology research is keep on working to build evidence to provide the perfect treatment for a particular patient. It is known as personalized medicine. However, personalized medicine is not free from death and competing risks of the patients. The general frame of a personalized medicine will always be compatible with close follow-up of the patients. Thus provide the requirement to develop the method towards joint longitudinal and survival modeling with competing risk modeling. This suggested method provides the scope to further work with joint longitudinal and survival modeling with competing risk analysis.

\section{Conflicts of interest}

The authors declare no conflict of interests.

\section{Acknowledgements}

The author thanks the two anonymous referees for their cautious reading and constructive suggestions which have led to improvement on earlier versions of the manuscript.

\section{References}

1. Cox DR. Regression models and life-tables. In: Kotz S, Johnson NL, eds. Breakthroughs in Statistics: Methodology and Distribution. New York, NY: Springer New York; 1992:527-541

2. Grambsch PM, Therneau TM, Fleming TR. Diagnostic plots to reveal functional form for covariates in multiplicative intensity models. Biometrics. 1995;51:1469-1482.

3. Schemper M, Henderson R. Predictive accuracy and explained variation in Cox regression. Biometrics. 2000;56:249-255

4. Chen K, Jin Z, Ying Z. Semiparametric Analysis of Transformation Models with Censored Data. vol 89. Biometrika: Oxford University Press; 2002:659-668.

5. Kalbfleisch JD, Prentice RL. The Statistical Analysis of Failure Time Data. John Wiley \& Sons; 2011.

6. Huang C-Y, Wang M-C. Joint modeling and estimation for recurrent event processes and failure time data. J Am Stat Assoc. 2004:99:1153-1165.

7. Chen M-H, Ibrahim JG, Sinha D. A new joint model for longitudinal and survival data with a cure fraction. J Multivar Anal. 2004;91:18-34.

8. Bhattacharjee A, Nath DC. Joint Longitudinal and Survival Data Modelling: An Application in Anti-diabetes Drug Therapeutic Effect. Statistics. ceeol.com; 2014 Available: https://www.ceeol.com/content-files/document-443635.pdf.

9. Chi Y-Y, Ibrahim JG. Joint models for multivariate longitudinal and multivariate survival data. Biometrics. 2006;62:432-445

10. Rizopoulos D, Ghosh P. A Bayesian semiparametric multivariate joint model for multiple longitudinal outcomes and a time-to-event. Stat Med. 2011;30:1366-1380.

11. Ding J, Wang J-L. Modeling longitudinal data with nonparametric multiplicative random effects jointly with survival data. Biometrics. 2008;64:546-556.

12. Robins J. Information recovery and bias adjustment in proportional hazards regression analysis of randomized trials using surrogate markers. Proceedings of the Biopharmaceutical Section. American Statistical Association; 1993:24-33.

13. Robins J. A new approach to causal inference in mortality studies with sustained exposure periods - application to control of the healthy worker survivor effect. Math Model. 1986;7:1393-1512.

14. Robins J. Lin D, Heagerty P, eds. Proceedings of the Second Seattle Symposium on Biostatistics. New York: Springer; 2004.

15. Robins J. Berkane M, ed. Latent Variable Modeling and Applications to Causality: Lecture Notes in Statistics. New York, NY: Springer-Verlag; 1997.

16. Robins J. Sechrest L, Freeman H, Mulley A, eds. Health Service Research Methodology: A Focus on AIDS. New York: NCHSR, U.S. Public Health Service; 1989

17. Murphy S. Optimal dynamic treatment regimes (with discussions) (Series B). J Roy Stat Soc. 2003:65:331-366.

18. Geskus RB. Cause-specific cumulative incidence estimation and the fine and gray model under both left truncation and right censoring. Biometrics. 2011;67:39-49.

19. Tsiatis A. A nonidentifiability aspect of the problem of competing risks. Proc Natl Acad Sci U S A. 1975;72:20-22.

20. Gray RJ. A class of K-sample tests for comparing the cumulative incidence of a competing risk. Ann Stat Inst Math Statistics. 1988;16:1141-1154.

21. Zeng D, Cai J. Simultaneous modelling of survival and longitudinal data with an application to repeated quality of life measures. Lifetime Data Anal. 2005;11:151-174.

22. Bhattacharjee A, Jose J, Vishwakarma GK. Joint modeling of missing and 
mismeasured measurements for computing radiotherapy margins. Clin Epidemiol Global Health. 2017;5(3):124-130.

23. Song X, Davidian M, Tsiatis AA. A semiparametric likelihood approach to joint modeling of longitudinal and time-to-event data. Biometrics. 2002;58:742-753.

24. Faucett CL, Thomas DC. Simultaneously modelling censored survival data and repeatedly measured covariates: a Gibbs sampling approach. Stat Med. 1996;15:1663-1685.

25. Xu J, Zeger SL. Joint analysis of longitudinal data comprising repeated measures and times to events. J R Stat Soc Ser C Appl Stat. 2001;50:375-387 Blackwell Publishers Ltd.

26. Tseng Y-K, Hsieh F, Wang J-L. Joint Modelling of Accelerated Failure Time and Longitudinal Data. vol 92. Biometrika: Oxford University Press; 2005:587-603.

27. Elashoff RM, Li G, Li N. A joint model for longitudinal measurements and survival data in the presence of multiple failure types. Biometrics. 2008;64:762-771.

28. Bhattacharyya $\mathrm{T}$, Bhattacharjee $\mathrm{A}$. Competing risk: an illustration with aspiration pneumonia in head and neck cancer patients undergoing radical radiotherapy: a biostatistician's perspective. Indian J Canc. 2014;51:406.

29. Bhattacharjee A. Bayesian competing risks model: an application to breast cancer clinical trial with incomplete observations. J Stat Manag Syst. 2015;18:381-404 Taylor \& Francis.

30. Murphy SA, van der Laan MJ, Robins JM, CPPRG. Marginal mean models for dynamic regimes. J Am Stat Assoc. 2001;96:1410-1423.

31. Murphy SA. Optimal dynamic treatment regimes. J R Stat Soc Series B Stat Methodol. 2003;65:331-355 Blackwell Publishing.

32. Robins JM. Optimal structural nested models for optimal sequential decisions. Proceedings of the Second Seattle Symposium in Biostatistics. New York, NY: Springer; 2004:189-326.

33. Robins J. A new approach to causal inference in mortality studies with a sustained exposure period-application to control of the healthy worker survivor effect. Math Model. 1986;7:1393-1512

34. May Watkins C. Learning from delayed rewards. Ph. D. thesis 1989; 1989 King's College, Cambridge, UK http://www.cs.rhul.ac.uk/ chrisw/new_thesis.pdf.

35. Christopher JC, Dayan P. Q-learning. Mach Learn. vol. 8. Kluwer Academic Publishers; 1992:279-292.
36. Murphy SA, Oslin DW, Rush AJ, Zhu J, MCATS. Methodological challenges in constructing effective treatment sequences for chronic psychiatric disorders. Neuropsychopharmacology. 2007;32:257-262.

37. Zhao Y-Q, Laber EB. Estimation of optimal dynamic treatment regimes. Clin Trials. 2014;11:400-407.

38. Qian M, Murphy SA. Performance guarantees for individualized treatment rules. Ann Stat. 2011;39:1180-1210.

39. Lunceford JK, Davidian M, Tsiatis AA. Estimation of Survival Distributions of Treatment Policies in Two-stage Randomization Designs in Clinical Trials. vol 58. Biometrics. Wiley Online Library; 2002:48-57.

40. Robins JM, Rotnitzky A, Zhao LP. Estimation of regression coefficients when some regressors are not always observed. J Am Stat Assoc. 1994;89:846-866 Taylor \& Francis

41. Guo X, Tsiatis A. A weighted risk set estimator for survival distributions in two-stage randomization designs with censored survival data. Int J Biostat. 2005;1. https://doi. org/10.2202/1557-4679.1000.

42. Scrucca L, Santucci A, Aversa F. Competing risk analysis using R: an easy guide for clinicians. Bone Marrow Transplant. 2007;40:381-387.

43. Austin PC, Lee DS, Fine JP. Introduction to the analysis of survival data in the presence of competing risks. Circulation. 2016;133:601-609.

44. Crowder MJ. Classical Competing Risks. CRC Press; 2001

45. Rao BR, Talwalker S, Kundu D. Confidence Intervals for the Relative Risk Ratio Parameter from Survival Data under a Random Censorship Model in Biomedical and Epidemiologic Studies. Biom J. Wiley Online Library; 1991 Available: http:// onlinelibrary.wiley.com/doi/10.1002/bimj.4710330808/full.

46. Sen A, Banerjee M, Li Y, Noone A-M. A Bayesian approach to competing risks analysis with masked cause of death. Stat Med. 2010;29:1681-1695.

47. Ge M, Chen M-H. Bayesian inference of the fully specified subdistribution model for survival data with competing risks. Lifetime Data Anal. 2012;18:339-363.

48. Basu S, Tiwari RC. Breast cancer survival, competing risks and mixture cure model: a Bayesian analysis. J Roy Stat Soc. 2010;173(2):307-329.

49. Fine JP, Gray RJ. A proportional hazards model for the subdistribution of a competing risk. J Am Stat Assoc. 1999;94:496-509 Taylor \& Francis. 